\title{
Prevalence of hibernating myocardium in patients with severely impaired ischaemic left ventricles
}

\author{
A Al-Mohammad, I R Mahy, M Y Norton, G Hillis, J C Patel, P Mikecz, S Walton
}

\begin{abstract}
Objective-Severe impairment of left ventricular (LV) contraction is associated with an adverse prognosis in patients with ischaemic heart disease. Revascularisation may improve the impaired $L V$ contraction if hibernating myocardium is present. The proportion of patients likely to benefit from this intervention is unknown. Therefore, the prevalence of hibernating myocardium in patients with ischaemic heart disease and severe impairment of $\mathrm{LV}$ contraction was assessed. Design-From a consecutive series of patients undergoing coronary angiography for the investigation of chest pain or LV impairment, all patients with ischaemic heart disease and an LV ejection fraction (LVEF) $\leqslant 30 \%$ were identified. These patients underwent positron emission tomography (PET) to detect hibernating myocardium, identified by perfusion metabolism mismatch.

Setting-A teaching hospital directly serving 500000 people.

Results-Of a total of 301 patients, 36 had ischaemic heart disease and an LVEF $\leqslant 30 \%$. Twenty-seven patients had PET images, while nine patients were not imaged because of emergency revascularisation (three), loss to follow up (one), inability to give consent (four), and age $<50$ years (one, ethics committee guidelines). Imaged and non-imaged groups were similar in $L V$ impairment, demographic characteristics, and risk factor profile. Fourteen patients $(52 \%$ of the imaged or $39 \%$ of all patients with ischaemic heart disease and LVEF $\leqslant 30 \%$ ) had significant areas of hibernating myocardium on PET.

Conclusion-It is possible that up to $50 \%$ of patients with ischaemic heart disease and severely impaired left ventricles have hibernating myocardium.

(Heart 1998;80:559-564)
\end{abstract}

Keywords: hibernating myocardium; left ventricular impairment; positron emission tomography

The Aberdeen PET Unit, Department of Bio-medical Physics and Bio-engineering, Aberdeen University, Aberdeen, UK

M Y Norton

P Mikecz

Correspondence to: Dr Al-Mohammad

Accepted for publication 12 August 1998

The importance of heart failure lies not only in its high prevalence, but also in the adverse prognosis associated with the diagnosis. The most common aetiology of impaired left ventricular (LV) contraction is ischaemic heart disease in which the degree of impairment is closely associated with outcome. ${ }^{1}$ For patients with severe heart failure, mortality rates may be as high as $60 \%$ at one year, ${ }^{2}$ while in those with moderate heart failure optimal medical therapy may improve survival to no better than $70 \%$ at two years. ${ }^{3}$ The three main randomised surgical studies of revascularisation in ischaemic heart disease have suggested that it is the patients with impaired LV contraction who have most to gain in survival terms from surgery. ${ }^{14-7}$ However, intervention in patients with severe LV impairment carries increased risk, particularly in patients with an LV ejection fraction (LVEF) lower than 35\% (a largely excluded subgroup from the main randomised surgical trials). Therefore, it is desirable to identify preoperatively those patients with poor LV contraction who are most likely to gain from surgery, thereby justifying their exposure to the increased perioperative risks.

Hibernating myocardium is the chronically ischaemic myocardium with impaired contraction, which improves with revascularisation. ${ }^{8}$ Preoperative identification of markers of hibernating myocardium has been shown to predict the recovery of regional and possibly global ventricular contraction after revascularisation..$^{9-15}$ On the other hand, non-viable myocardium does not regain contraction with revascularisation. Identifying markers of hibernating myocardium in patients with severely impaired LV contraction preoperatively means, therefore, that only patients with most to gain need be exposed to the risks of surgery.

The proportion of patients with severe impairment of LV contraction who have hibernating myocardium remains unknown. However, Bonow estimates from several clinical series that between $25 \%$ and $40 \%$ of patients with chronic coronary artery disease and global LV impairment have the potential for significant improvement in LVEF after revascularisation. ${ }^{16}$ From that, the prevalence of hibernating myocardium in patients with ischaemic LV impairment was extrapolated. These series were affected by preselection bias, since they reported the incidence of hibernating myocardium in patients undergoing viability studies and revascularisation. There are, however, no preoperative studies of the true prevalence of hibernating myocardium in patients with poor LV contraction.

By definition the presence of hibernating myocardium can only be ascertained retrospectively, by demonstrating an improvement in LV contraction after revascularisation. Given the high risk of operating on patients with poor LV contraction, and the fact that only those with hibernating myocardium among the latter group would benefit from surgery, it is unethical to subject all patients with poor LV contraction to revascularisation to establish the 
true prevalence of hibernating myocardium. Therefore, surrogate markers for hibernating myocardium could be studied preoperatively to assess indirectly the prevalence of the phenomenon in this high risk patient group. Of several techniques available to predict the presence of hibernating myocardium preoperatively, demonstrating areas of hypoperfused myocardium with increased glucose uptake, reduced perfusion at rest, and impaired contraction by positron emission tomography (PET), is widely accepted as the "gold standard". ${ }^{12} 13$

We have, therefore, used PET to study the prevalence of hibernating myocardium in a consecutive series of patients undergoing coronary angiography, who have coronary artery disease and severely impaired left ventricles.

\section{Methods}

SUBJECTS

A consecutive series of patients with ischaemic heart disease and severely impaired LV contraction were prospectively identified as follows.

All patients undergoing coronary angiography for the investigation of chest pain or LV impairment under the care of one senior investigator between March and October 1995 were considered for inclusion in the study ( $\mathrm{n}=301$, 204 male). This was carried out in a teaching hospital directly serving a population base of 500 000. Therefore, the patients' population base is representative of the general population and is not affected by the preselection bias that normally affects series from tertiary referral centres. From this cohort of 301 patients, all subjects fulfilling the following angiographic entry criteria were prospectively identified: (a) presence of severe stenosis in at least one major epicardial coronary artery; (b) LVEF $\leqslant 30 \%$ as measured by the modified Sandler and Dodge Area-Length method, ${ }^{17}$ using a single plane contrast left ventriculogram in the right anterior oblique projection. (This measure of "severe" LV impairment was chosen because below this level subjects would not have been randomised into the major surgical trials).

Coronary artery disease was present in 260 patients (188 male) of whom 36 had an LVEF $\leqslant 30 \%$.

WALL MOTION ANALYSIS

LV contraction was further assessed by assignment of a wall motion score. Two experienced cardiologists, blinded to whether the patient was imaged, divided the LV outline in the right anterior oblique projection of the left ventriculogram into five segments: antero-basal; antero-apical; apical; infero-apical; and inferobasal. Each segment was scored as follows: normal 1; hypokinetic 2 ; severely hypokinetic 3 ; akinetic 4; and dyskinetic 5. This gives a normal LV wall motion score of 5 . The two observers agreed on the scoring of 80 segments, whereas they differed by not more than 1 scoring grade in 50 segments (variance $\mathrm{S} 2=0.097$, coefficient of variance $=0.1$ ). One female patient did not undergo contrast LV angiography because of the presence of intraventricular thrombosis; her LVEF was measured on transthoracic echocardiography.
ETHICS COMMITTEE APPROVAL

All patients gave written informed consent before they had PET imaging. The protocol for this research study was approved by the local research ethics committee. It was a condition of approval that only subjects over the age of 50 years should be studied. One 47 year old male patient, however, underwent PET imaging for clinical reasons, and his data were included as he was part of the total cohort of patients with poor LV contraction.

ASSESSMENT OF HIBERNATION

The presence of hibernation was detected by PET, based on the assessment of metabolism and flow using two different tracers. ${ }^{13} \mathrm{~N}-$ ammonia $\left({ }^{13} \mathrm{~N}-\mathrm{NH}_{3}\right)$ was used to study the regional myocardial perfusion. ${ }^{18-22}{ }^{18} \mathrm{~F}-2$ fluoro2-deoxyglucose $\left({ }^{18} \mathrm{~F}\right.$-FDG) was used to differentiate between viable myocardium and scar tissue. ${ }^{1323}$

Tomographic imaging was performed using a Siemens Exact 31 PET scanner (CTI PET Systems Inc, Knoxville, Tennessee, USA). This system produces 31 slices, with a slice separation of $3 \mathrm{~mm}$ resulting in a volume image $10.6 \mathrm{~cm}$ deep. Reconstructed image resolution is $10 \mathrm{~mm}$. Attenuation correction was achieved by performing a transmission scan using three revolving rod sources before the emission scan.

For each subject, a mean (SD) of $11.2(5.2) \mathrm{mCi}$ of ${ }^{13} \mathrm{~N}-\mathrm{NH}_{3}$ was given intravenously and volume images of myocardial uptake gathered up to 20 minutes after injection. On the same day, a mean (SD) of $4.8(0.5) \mathrm{mCi}$ of ${ }^{18} \mathrm{~F}-$ FDG was injected intravenously one hour after the patient had received $50 \mathrm{~g}$ of oral glucose. ${ }^{24}$ Diabetic patients received intravenous soluble insulin at a dose of 4-10 units, according to their serum glucose concentration. The images were acquired 60 minutes postinjection. The ${ }^{18} \mathrm{~F}$ FDG emission scan was gathered in ECG gated mode using the $\mathrm{R}$ wave on the ECG in all patients who were in sinus rhythm. ${ }^{25}$

For regional wall motion analysis by gated FDG, we assessed wall thickening in the following fashion.

Myocardial uptake was measured by reforming the ventricle into four short axis sections covering the base to mid-ventricle. The apex to mid-ventricle was reformed as eight long axis sections at $22.5^{\circ}$ intervals round the ventricle's long axis. Radial profiles were formed at $22.5^{\circ}$ intervals round each short axis section producing 16 profiles for each short axis section. Five radial profiles were formed at $30^{\circ}$ intervals from each of the long axis sections. This three dimensional sampling profile set more closely reflects the most likely wall thickening direction than simple short axis sampling. A complete set of wall profiles was obtained at each of eight phases using a fixed laboratory frame of reference.

Each profile was analysed to obtain a hybrid wall thickness parameter given by the product of maximum wall uptake in the profile and the "thickness" of the wall as given by the second moment of the radial profile. Use of the second moment of radial profile allows for partial volume effects. ${ }^{26}$ The product of uptake and 
A
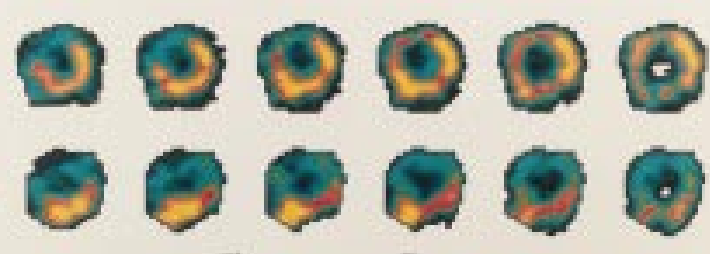

C

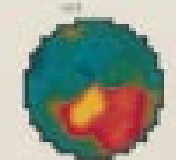

D)
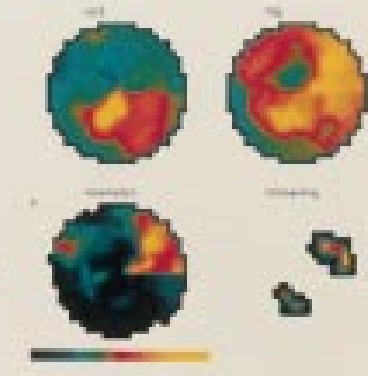

Figure 1 PET images of a 73 year old man with multivessel coronary artery disease and severe LV impairment. Row A represents the metabolic tomographic images after ${ }^{18} \mathrm{~F}-F D G$ injection. Row $B$ shows the perfusion tomographic images after ${ }^{13} \mathrm{~N}-\mathrm{NH}_{3}$ injection. Row $\mathrm{C}$ shows the perfusion polar map on the left and the metabolic polar map on the right. Row D shows the mismatch polar map on the left and the wall thickening polar map on the right. This figure represents an important area of perfusion metabolism mismatch (hibernating myocardium) in the anterolateral area.

Table 1 A comparison between the imaged and non-imaged patients

\begin{tabular}{llll}
\hline Characteristics & $\begin{array}{l}\text { Imaged patients } \\
\left(n_{1}=27\right)\end{array}$ & $\begin{array}{l}\text { Non-imaged patients } \\
\left(n_{2}=9\right)\end{array}$ & Significance \\
\hline Mean (SD) age (years) & $63.4(9.3)$ & $64.4(11.4)$ & NS \\
Male sex & $22 / 27(81.5 \%)$ & $7 / 9(77.8 \%)$ & NS \\
Mean (SD) stenosed coronary arteries ${ }^{\star}$ & $2.3(0.8)$ & $2.7(0.7)$ & NS \\
Mean (SD) LV score† & $13.9(2.2)$ & $12.7(1.9)$ & NS \\
Mean (SD) LVEDP (mm Hg) $\neq$ & $13.5(8)$ & $13.4(7)$ & NS \\
Smoking\$ & $23 / 27(85.2 \%)$ & $7 / 9(77.8 \%)$ & NS \\
Hypercholesterolaemia & $26 / 27(96.3 \%)$ & $7 / 9(77.8 \%)$ & NS \\
Diabetes & $3 / 27(11.1 \%)$ & $1 / 9(11.1 \%)$ & NS \\
Hypertension†† & $4 / 27(14.8 \%)$ & $3 / 9(33.3 \%)$ & NS \\
\hline
\end{tabular}

NS, not significant (significance is when $\mathrm{p}<0.05$ ); ${ }^{\star}$ Number of epicardial coronary arteries with stenosis $>70 \%$; $+\mathrm{LV}$ score, the sum of scores given for wall motion to the five segments of the left ventricle on the RAO projection of the LV angiogram; $¥$ LVEDP, left ventricular end diastolic pressure, measured through an intraventricular pigtail catheter at the time of the cardiac catheterisation; \$Smoking, patients who are current smokers or exsmokers; $\uparrow$ Hypercholesterolaemia, defined as total serum cholesterol $>5.2 \mathrm{mmol} / 1$; ${ }^{\star \star}$ Diabetes, both insulin dependent and non-insulin dependent diabetes mellitus according to World Health Organisation criteria irrespective of the treatment modality; ++ Hypertension, either blood pressure $>140 / 90 \mathrm{~mm} \mathrm{Hg}$ at the time of catheterisation or in the past, whether on treatment.

apparent thickness has been shown to be more linearly related to actual wall thickness than the individual parameters. ${ }^{27}$ The time variation in hybrid wall thickness for each profile direction was Fourier analysed to obtain an amplitude and phase value. The amplitude was subsequently converted into a percentage wall thickening value. The variation in wall thickening was either graphically displayed or converted to a polar map representation.

The reconstructed images were rotated and

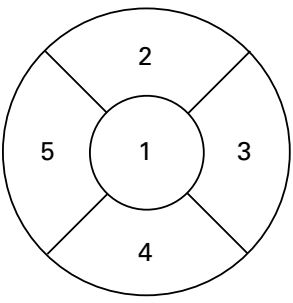

Figure 2 A polar map of the LV myocardium, divided into five regions: apical (1); anterior (2); lateral (3); inferior (4); and septal (5). resliced to generate short axis sections of the myocardium which were subsequently analysed using circumference profile analysis to produce polar maps diagrammatically showing the myocardial distribution of the tracers (fig 1). ${ }^{26}$ The left ventricle, as represented by the polar map, was divided into five regions (fig 2): apical; anterior; lateral; inferior; and septal. These were different from the segments studied on the right anterior oblique projection of the LV angiogram for wall motion scoring.

The myocardial region with the highest uptake of ${ }^{13} \mathrm{~N}-\mathrm{NH}_{3}$, was taken as normal
${ }^{13} \mathrm{~N}-\mathrm{NH}_{3}$ concentration for that patient. The abnormally perfused areas were semiquantitatively compared to the reference "normal" area. The glucose uptake in that normalised area was regarded as the normal ${ }^{18} \mathrm{~F}-\mathrm{FDG}$ uptake. The rest of the myocardium was then compared to this reference concentration.

Areas of the myocardium were defined as suggestive of hibernation if ${ }^{13} \mathrm{~N}-\mathrm{NH}_{3}$ uptake was reduced, ${ }^{18} \mathrm{~F}$-FDG uptake was increased, and contraction reduced (mismatched defect). ${ }^{24}$ The assessment of mismatch was carried out qualitatively by simultaneous inspection of perfusion and metabolism images. The inspection was carried out independently by two observers whose conclusions were concordant in $92 \%$ of the cases. The differences were resolved by consensus. The patient was regarded as having hibernating myocardium if the mismatch defect affected a predetermined arbitrary cut off point which equals $20 \%$ of the LV mass.

Details of the coronary artery anatomy were related to the LV map, using the distribution of the arteries as shown in the projection closest to that used to produce the polar map, namely the left anterior oblique projection with caudal tilt.

STATISTICS

Continuous variables are presented as mean (SD). Categorical data are presented as frequencies. Imaged and non-imaged groups were compared using Student's $t$ test for independent samples, with the exception of coronary risk factors, which were compared using $\chi^{2}$ test for categorical data. Results are expressed as mean (SD). Significance was decided at $\mathrm{p}<0.05$. Among the imaged patients, the same statistical methods were applied when comparing patients with and without hibernating myocardium.

Data were analysed using SPSS for Microsoft Windows Release 6.1 software package.

\section{Results}

Of 36 patients fulfilling the entry criteria, 27 (23 men) underwent successful PET imaging. Nine patients were not imaged because of emergency revascularisation (three), loss to follow up (one), refusal or inability to give consent (four), and age $<50$ years (one, ethics committee guidelines).

Imaged and non-imaged patients were similar for demographic characteristics, risk factor profile, the number of significantly stenosed or occluded coronary arteries, and LV wall motion score (table 1). However, one third of the non-imaged patients had Canadian Cardiovascular Society (CCS) class IV angina, whereas the CCS class of all imaged patients was less than class IV.

The significance of coronary artery stenosis was assessed by eyeballing, and the stenosis was significant if it was $\geqslant 70 \%$. Smokers were defined as current or exsmokers, whereas nonsmokers were the patients who were never actively exposed to smoking. Hypercholesterolaemia was defined as a total serum cholesterol concentration $\geqslant 5.2 \mathrm{mmol} / 1$. Patients were considered hypertensive if they had a systolic blood pressure $>140 \mathrm{~mm} \mathrm{Hg}$ and/or a diastolic blood 
Table 2 Imaged patients detailed characteristics

\begin{tabular}{|c|c|c|c|c|c|c|}
\hline Patient & Age (years) & Sex & Mismatch & $C A D$ & $W M S$ & $E F(\%)$ \\
\hline 1 & 53 & Male & Yes & LAD & 12.5 & 20 \\
\hline 2 & 58 & Male & No & LAD & 12.5 & 30 \\
\hline 3 & 72 & Male & No & LAD, RCA, CX & 15.5 & 10 \\
\hline 4 & $(47)$ & Male & No & LAD, RCA, CX & 15.5 & 17 \\
\hline 5 & 52 & Male & Yes & LAD, RCA & 14 & 24 \\
\hline 6 & 76 & Female & No & LAD & 14 & 16 \\
\hline 7 & 64 & Male & Yes & LAD, RCA, CX & 19 & 17 \\
\hline 8 & 67 & Female & Yes & LAD, RCA, CX & 14.5 & 17 \\
\hline 9 & 67 & Male & No & LAD & 16 & 12 \\
\hline 10 & 87 & Male & No & LAD, RCA, CX & 13 & 21 \\
\hline 11 & 61 & Male & Yes & LAD, RCA & 11 & 27 \\
\hline 12 & 56 & Female & No & $\mathrm{LAD}, \mathrm{RCA}$ & - & 30 \\
\hline 13 & 64 & Female & No & LAD, RCA, CX & 11.5 & 20 \\
\hline 14 & 74 & Male & Yes & LAD, RCA, CX & 12.5 & 18 \\
\hline 15 & 55 & Female & No & LAD, CX & 17 & 11 \\
\hline 16 & 50 & Male & No & LAD, RCA & 17 & 10 \\
\hline 17 & 63 & Male & No & $\mathrm{RCA}$ & 12.5 & 19 \\
\hline 18 & 73 & Male & Yes & LAD, RCA, CX & 14.5 & 11 \\
\hline 19 & 64 & Male & Yes & LAD, RCA, CX & 10 & 16 \\
\hline 20 & 71 & Male & Yes & LAD, RCA, CX & 16 & 19 \\
\hline 21 & 67 & Male & No & RCA, CX & 14 & 20 \\
\hline 22 & 64 & Male & No & LAD, RCA & 15.5 & 17 \\
\hline 23 & 53 & Male & Yes & LAD, RCA, CX & 12.5 & 13 \\
\hline 24 & 58 & Male & Yes & LAD, RCA, CX & 13 & 30 \\
\hline 25 & 63 & Male & No & RCA, CX & 10.5 & 20 \\
\hline 26 & 57 & Male & Yes & LAD, RCA, CX & 13 & 13 \\
\hline 27 & 76 & Male & Yes & LAD, RCA, CX & 14 & 23 \\
\hline
\end{tabular}

CAD, coronary artery disease (> 70\% stenosis); LAD, left anterior descending artery; RCA, right coronary artery; CX, Circumflex artery; WMS, wall motion score; EF, ejection fraction.

Table 3 A comparison between the patients with and without hibernating myocardium

\begin{tabular}{|c|c|c|c|}
\hline Characteristics & $\begin{array}{l}\text { Patients with } \\
\text { hibernating } \\
\text { myocardium } \\
\left(n_{1}=14\right)\end{array}$ & $\begin{array}{l}\text { Patients without } \\
\text { hibernating } \\
\text { myocardium } \\
\left(n_{2}=13\right)\end{array}$ & Significance \\
\hline Mean (SD) age (years) & $63.3(8.1)$ & $63.5(10.8)$ & NS \\
\hline Male sex & $13 / 14(93 \%)$ & $9 / 13(69 \%)$ & NS \\
\hline Mean (SD) stenosed coronary arteries* & $2.6(0.8)$ & $2.1(0.8)$ & NS \\
\hline Mean (SD) LV score $†$ & $13.5(2.3)$ & $14.2(2.1)$ & NS \\
\hline Mean (SD) LVEDP $(\mathrm{mm} \mathrm{Hg}) \ddagger$ & $13.7(9.5)$ & $13.3(6.3)$ & NS \\
\hline Smoking $\sqrt{ }$ & $13 / 14(93 \%)$ & $10 / 13(77 \%)$ & NS \\
\hline Hypercholesterolaemia & $14 / 14(100 \%)$ & $12 / 13(92 \%)$ & NS \\
\hline Diabetes $^{\star \star}$ & $2 / 14(14 \%)$ & $1 / 13(8 \%)$ & NS \\
\hline Hypertension†† & $4 / 14(29 \%)$ & $0 / 13(0 \%)$ & $\mathrm{p}<0.05$ \\
\hline
\end{tabular}

NS, not significant (significance is when $\mathrm{p}<0.05$ ); ${ }^{\star}$ Number of epicardial coronary arteries with stenosis $>70 \%$; $\nmid \mathrm{LV}$ score, the sum of scores given for wall motion to the five segments of the left ventricle on the RAO projection of the LV angiogram; $¥$ LVEDP, left ventricular end diastolic pressure, measured through an intraventricular pigtail catheter at the time of the cardiac catheterisation; \$Smoking, patients who are current smokers or exsmokers; $₫$ Hypercholesterolaemia, defined as total serum cholesterol $>5.2 \mathrm{mmol} / 1$; ${ }^{\star \star}$ Diabetes, both insulin dependent and non-insulin dependent diabetes mellitus according to World Health Organization criteria irrespective of the treatment modality; ++ Hypertension, either blood pressure $>140 / 90 \mathrm{~mm} \mathrm{Hg}$ at the time of catheterisation or in the past, whether on treatment.

pressure $>90 \mathrm{~mm} \mathrm{Hg}$, or if they were on treatment for hypertension. Diabetics were considered together irrespective of their diabetes type or their treatment's modality.

The detailed characteristics of the imaged patients are presented in table 2 .

Of the 27 patients with images suitable for analysis, 14 had at least one region on the polar map fulfilling the PET criteria for hibernation. Thus, 14 of $27(52 \%)$ of the imaged patients and at least 14 of $36(39 \%)$ of the total cohort demonstrated evidence of hibernating myocardium. There were no statistically significant differences between patients with and without hibernating myocardium, except for hypertension, which affected four of the 14 patients with hibernating myocardium and none of the patients with no hibernating myocardium $(\mathrm{p}<0.05)($ table 3$)$.

\section{Discussion}

These data provide an estimate of the prevalence of hibernating myocardium in a consecutive series of patients with poor LV contraction using the best surrogate marker for the preoperative detection of hibernating myocardium. It appears that $50 \%$ of patients 50 years and older, with an LVEF $\leqslant 30 \%$, have the potential for improvement in LV contraction following revascularisation.

Although hibernating myocardium is defined by its recovery following revascularisation, a true estimate of prevalence cannot be defined in this way because not all patients have an indication for coronary artery bypass surgery on conventional grounds. Indeed, many patients are denied coronary artery bypass surgery because the risks of the operation are perceived to be excessive owing to their poor LV contraction. Of the currently available preoperative predictors of hibernation, PET has the best predictive accuracy and provides the best surrogate marker for hibernating myocardium to be predicted. Therefore, the mismatch pattern on PET was used in this report as a surrogate for hibernating myocardium.

The principles of the diagnosis of hibernating myocardium by PET lie in the biochemical behaviour of the ischaemic myocardium. The myocardium is unable to metabolise anaerobically long chain free fatty acids, which are its main source (approximately $70 \%$ ) of energy under normal conditions. Therefore, under ischaemic conditions, the myocardium will rely increasingly on the anaerobic metabolism of glucose as its main energy source, although the hibernating myocardium's metabolism is not exclusively anaerobic. ${ }^{28}$ Given these changes, the PET marker for hibernating myocardium is the mismatch between hypoperfusion of the region of interest and normal or increased uptake of the extrinsic glucose analogue ${ }^{18} \mathrm{~F}-\mathrm{FDG}$ in that region. This pattern is labelled as perfusion metabolism mismatch. ${ }^{12} 1329$ The mismatch pattern has a high positive and negative predictive accuracy of functional improvement with myocardial revascularisation. ${ }^{2} 121330$ The predictive value of PET is highest in the myocardial regions with severely impaired contraction and a mismatch pattern. ${ }^{13}$

Several investigators suggested the use of the hyperinsulinaemic euglycaemic glucose clamp technique while studying myocardial glucose metabolism, particularly in patients with coronary artery disease, who are known to have a high incidence of insulin resistance. However, the great majority of PET groups use our technique of oral glucose loading with bolus insulin doses to keep blood glucose under control. The reason behind the latter approach, other than simplicity, is that investigators have demonstrated that the use of glucose clamp improves the image quality of the glucose uptake map, but fails to produce clinically different conclusions compared to images produced using our approach. ${ }^{31}$

In the current study, 36 of 260 patients $(13.8 \%)$ with coronary artery disease were shown to have severely impaired LV contraction. This is similar to published estimates of those who require myocardial viability in clinical practice, which range between $10 \%$ and $20 \%{ }^{32}$ However, the prevalence of the best marker for hibernation was greater than previous estimates 
have suggested might be the case. Although it is possible that the group of patients not imaged may have skewed the results, even if none of these patients had evidence of hibernating myocardium the prevalence would be $39 \%$ (14 of 36). Such a bias is unlikely, given the similarity of the imaged and non-imaged groups. The two groups were comparable in all aspects except for the CCS angina class.

We are unaware of any data to suggest that the presence of angina at rest would affect the presence of hibernating myocardium in a patient with severely impaired LV contraction. It would be reasonable, therefore, to suggest that the findings in the imaged group are applicable to the total cohort of patients with severe impairment of LV contraction.

As for the comparison of the risk factors profile and demographic data between the patients with hibernating myocardium and those with no hibernating myocardium, significantly more hypertensive patients were present in the group with hibernating myocardium compared to those without. However, clinically important conclusions cannot be drawn from this observation, as we did not set out to investigate the differences between patients with and without hibernating myocardium. This is an observational study to investigate the prevalence of a phenomenon rather than to detect its contributing factors.

There is no consensus as to how much of the LV myocardium should be hibernating for a significant improvement to be expected with revascularisation. However, the figure of $20 \%$ has been quoted. ${ }^{183}$ We have used this level as a cut off point in deciding the threshold at which the patient is said to have hibernating myocardium. This, however, is an arbitrary point. We recognise that setting a different threshold could result in a different prevalence level.

The institution where this study was carried out is unique in that it is the only hospital in the country which has access to a PET facility, while directly serving the community, rather than relying on other institutions for referral. Therefore, this series is less likely to have been affected by the preselection bias that affects the series from tertiary referral centres.

The mortality of patients with coronary artery disease and severely impaired LV contraction is high, even on optimal medical therapy. ${ }^{2}$ However, revascularisation can lead to improved symptoms and prognosis in patients with impaired LV contraction as demonstrated in the surgical studies. ${ }^{211} 2934$

Given the risks of revascularisation in these patients, the preoperative identification of viable myocardium in patients with poor LV contraction would define the group of patients who stand to benefit most from revascularisation, justifying the risk of the operation. ${ }^{2} 7$ Furthermore, in addition to predicting improvement after revascularisation, there is evidence that the PET pattern of mismatch identifies a group of patients who are at very high risk for cardiac death if they were assigned to medical therapy alone. ${ }^{27}$ Our data from a consecutive series suggest that as many as $50 \%$ of patients with severely impaired LV contraction could benefit from revascularisation. This finding has important implications for the investigation and management strategies in this group of patients with poor prognosis.

This study was performed using a research grant from the Aberdeen Royal Hospitals National Health Service Trust's Endowment Fund, for which the authors express their gratitude.

1 Maddahi J, Schelbert H, Brunken R, et al. Role of thallium201 and PET imaging in evaluation of myocardial viability and management of patients with coronary artery disease and left ventricular dysfunction [review]. I Nucl Med 1994;35:707-15.

2 Packer M. Physiologic determinants of survival in congestive heart failure. Prolonging life in patients with congestive heart failure: the next frontier. Circulation 1987;75:IV-1-3.

3 The CONSENSUS Trial Study Group. Effects of enalapril on mortality in severe congestive heart failure; results of the mortality in severe congestive heart failure; results of the co-operative north Scandinavian enalapril survival
(CONSENSUS). N Engl F Med 1987;316:1429-35.

4 Murphy ML, Hultgren HN, Detre K, et al. Treatment of Murphy ML, Hultgren HN, Detre K, et al. Treatment of
chronic stable angina. A preliminary report of survival data of the randomized Veterans Administration co-operative of the randomized Veterans Admini
study. N Engl F Med 1977;297:621-7.

5 The European Coronary Surgery Study Group. Prospective randomised study of coronary artery bypass surgery in stable angina pectoris. Second interim report. Lancet 1980; ii:491-5.

6 CASS Principal Investigators and their Associates. Coronary artery surgery study (CASS): a randomized trial of coronary bypass surgery. Survival data. Circulation 1983;68: 939-50

7 Eitzman D, Al-Aouar Z, Kanter HL, et al. Clinical outcome of patients with advanced coronary artery disease after viability studies with positron emission tomography. $7 \mathrm{Am}$ Coll Cardiol 1992;20:559-65.

8 Rahimtoola SH. The hibernating myocardium. Am Heart $\mathcal{F}$ 1989;117:211-21

9 Tillisch J, Brunken R, Marshall R, et al. Reversibility of cardiac wall-motion abnormalities predicted by positron tomdiac wall-motion abnormalities predicted

10 Tamaki N, Yonekura Y, Yamashita K, et al. Positron emission tomography using fluorine-18 deoxyglucose in evaluation of coronary artery bypass grafting. Am $\mathcal{F}$ Cardiol 1989;64:860-5

11 Nienaber CA, Brunken RC, Sherman CT, et al. Metabolic and functional recovery of ischaemic human myocardium after coronary angioplasty. $\mathcal{F}$ Am Coll Cardiol 1991;18:96678 .

12 Marwick TH, MacIntyre WJ, Lafont A, et al. Metabolic responses of hibernating and infarcted myocardium to revascularization. A follow-up study of regional perfusion, function, and metabolism. Circulation 1992;85:1347-53.

13 vom Dahl J, Eitzman DT, Al-Aouar ZR, et al. Relation of regional function, perfusion, and metabolism in patients with advanced coronary artery disease undergoing surgical revascularization. Circulation 1994;90:2356-66.

14 akeishi Y, Tono-oka I, Kubota I, et al. Functional recovery of hibernating myocardium after coronary bypass surgery: hibernating myocardium after coronary bypass surgery: f 1991;122:665-70.

15 Marwick TH, Nemec JJ, Lafont A, et al. Prediction by postexercise fluoro-18-deoxyglucose positron emission tomography of improvement in exercise capacity after revscularization. Am f Cardiol 1992;69:854-9.

16 Bonow RO. Identification of viable myocardium [editorial]. Circulation 1996;94:2674-80.

17 Sandler H, Dodge HT. The use of single plane angiocardiograms for the calculation of left ventricular volume in man. Am Heart F 1968;75:325-34.

18 Chan RK, Lee KJ, Calafiore P, et al. Comparison of dobutamine echocardiography and positron emission tomography in patients with chronic ischemic left ventricular dysfunction. $\mathcal{F}$ Am Coll Cardiol 1996;27:1601-7.

19 Schelbert HR, Phelps ME, Huang SC, et al. N-13 ammonia as an indicator of myocardial blood flow. Circulation 1981; 63:1259-72.

20 Hutchins GD, Schwaiger M, Rosenspire KC, et al. Noninvasive quantification of regional blood flow in the human heart using $\mathrm{N}-13$ ammonia and dynamic positron emission tomographic imaging. F Am Coll Cardiol 1990;15:1032-42.

21 Schelbert HR, Phelps ME, Hoffman EJ, et al. Regional myocardial perfusion assessed with $\mathrm{N}-13$ labelled ammonia and positron emission computerized axial tomography. $\mathrm{Am}$ f Cardiol 1979;43:209-18.

22 Bergmann SR, Hack S, Tewson T, et al. The dependence of accumulation of ${ }^{13} \mathrm{NH} 3$ by the myocardium on metabolic factors and its implications for quantitative assessment of perfusion. Circulation 1980;61:34-43.

23 Georg Wolpers H, Burchert W, van den Hoff J, et al. Assessment of myocardial viability by use of $11 \mathrm{C}$-acetate and positron emission tomography, threshold criteria of reversible dysfunction. Circulation 1997;95:1417-24.

24 Adams JN, Norton $M$, Trent RJ, et al. Incidence of hibernating myocardium after acute myocardial infarction hibernating myocardium after acute myocardial inf

25 Yamashita K, Tamaki N, Yonekura Y, et al. Regional wall thickening of left ventricle evaluated by gated positron emission tomography in relation to myocardial perfusion and glucose metabolism. $\mathcal{F} \mathrm{Nucl} \mathrm{Med} \mathrm{1991;32:679-85.}$ 
26 Goris ML, Thomson C, Malone LJ, et al. Modelling the integration of myocardial regional perfusion and function. Nucl Med Comm 1994;15:9-20.

27 Buvat I, Bartlett ML, Kitsiou AN, et al. A "hybrid" method for measuring myocardial wall thickening from gated PET/ SPECT images. F Nucl Med 1997;38:324-9.

28 Gropler RJ, Geltman EM, Sampathkumaran K, et al. Functional recovery after coronary revascularization for chronic
coronary artery disease is dependent on maintenance of oxidative metabolism. f Am Coll Cardiol 1992;20:569-77.

29 Schelbert HR. Positron emission tomography for the assessment of myocardial viability. Circulation 1991;84:I-122-31.

30 Tamaki N, Yonekura Y, Yamashita K, et al. Prediction of reversible ischemia after coronary artery bypass grafting by positron emission tomography. Am f Cardiol 1991;21:193201.
31 Knuuti MJ, Nuutila P, Ruotsalainen U, et al. Euglycemic hyperinsulinemic clamp and oral glucose load in stimulating myocardial glucose utilization during positron emission

32 Lemlek J, Heo J, Iskandrian AS. The clinical relevance of myocardial viability in patient management. Am Heart $\mathcal{F}$ 1992;124:1327-31.

33 Di Carli MF, Asgarzadie F, Schelbert HR, et al. Quantitative relation between myocardial viability and improvement in heart failure symptoms after revascularization in patients with ischemic cardiomyopathy. Circulation 1995;92:343644.

34 Yoshida K, Gould KL. Quantitative relation of myocardial infarct size and myocardial viability by positron emission tomography to left ventricular ejection fraction and 3-year mortality with and without revascularization. $\mathrm{f} \mathrm{Am} \mathrm{Coll}$ Cardiol 1993;22:984-97.

\section{STAMPS IN CARDIOLOGY}

\section{Heart foundations on stamps (1)}

New Zealand has issued health stamps each year since 1929. These stamps always bear a charity surcharge and were originally used to support health camps for children. A set of two health stamps was issued on 2 August 1978. The first commemorates the 50th anniversary of health stamp issues, and the original stamp from 1929 has been reproduced in the design. There were two versions of the 1929 stamp, which are identical apart from the inscription. The original stamp was inscribed "Help stamp out

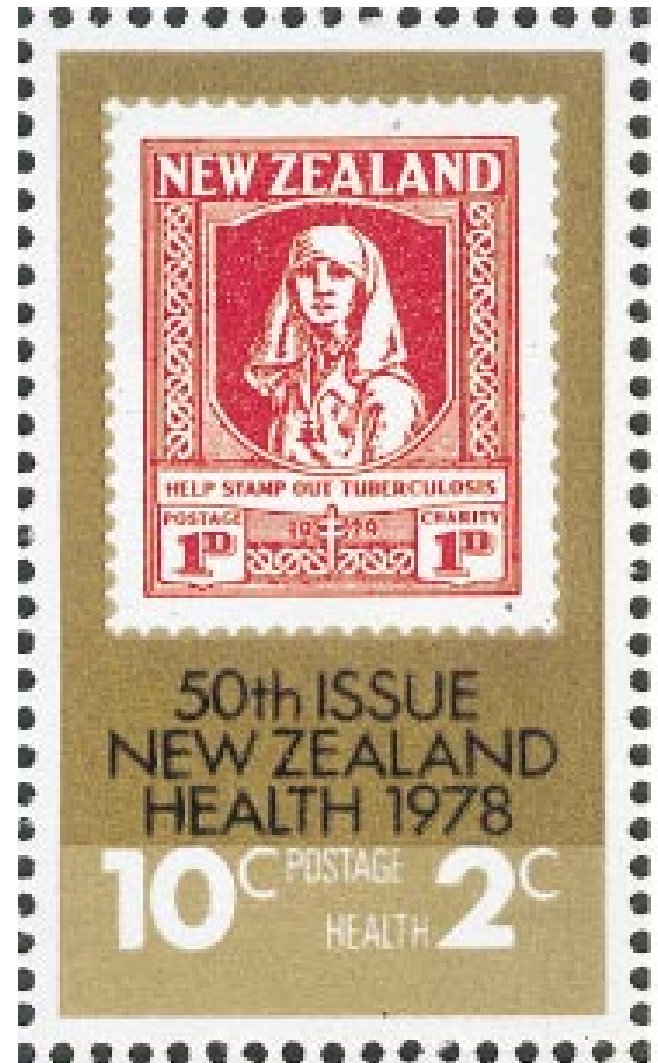

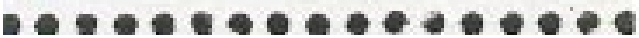

tuberculosis" and the later issue "Help promote health". The second 1978 health stamp, depicting cardiac surgery, commemorates the 10th anniversary of the formation of the National Heart Foundation of New Zealand, which was incorporated under the provisions of the Charitable Trusts Act on 26 April 1968. The emblem of the National Heart Foundation of New Zealand is incorporated in the lower left corner of the main stamp design. These stamps were on sale for nearly a year until 30 June 1979 .

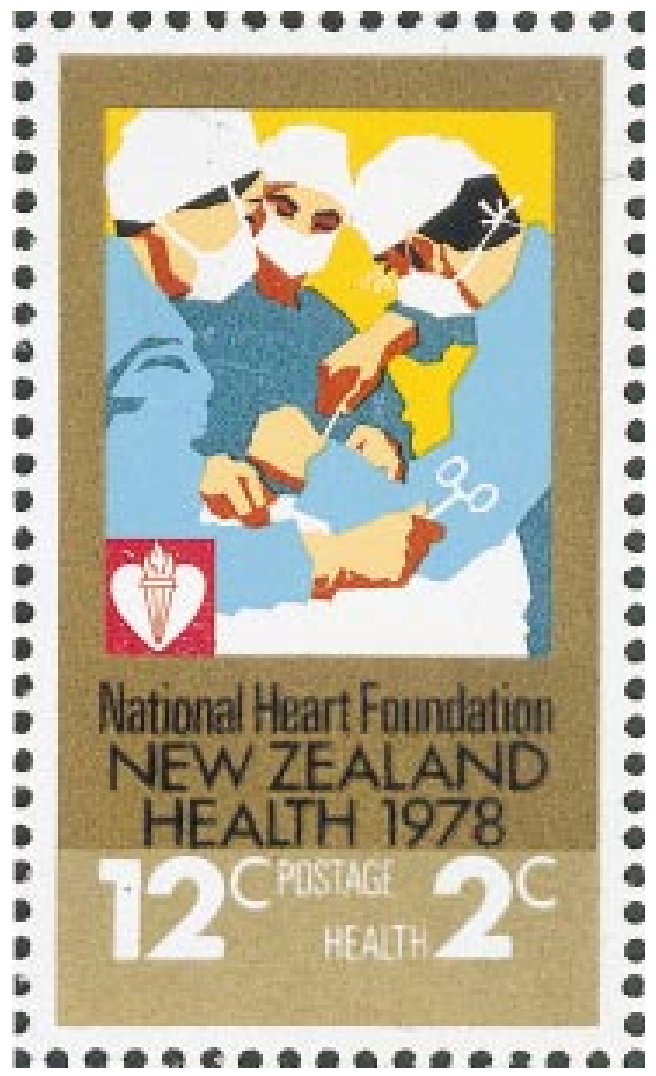

M K DAVIES A HOLLMAN 